\title{
ON ELASTIC WAVES IN A THINLY-LAYERED LAMINATED MEDIUM WITH STRESS COUPLES UNDER INITIAL STRESS
}

\author{
P. PAL ROY \\ Blasting Department \\ Central Mining Research Station \\ Barwa Road, Dhanbad 826001 INDIA \\ and

\section{DEBNATH} \\ Department of Mathematics \\ University of Central Florida \\ Orlando, FL 32816, U.S.A.
}

(Received September 9, 1985 and in revised form January 30, 1987)

ABSTRACT: The present work is concerned with a simple transformation rule in finding out the composite elastic coefficients of a thinly layered laminated medium whose bulk properties are strongly anisotropic with a microelastic bending rigidity. These elastic coefficients which were not known completely for a layered laminated structure, are obtained suitably in terms of initial stress components and Lame's constants $\lambda_{i}, \mu_{i}$ of initially isotropic solids. The explicit solutions of the dynamical equations for a prestressed thinly layered laminated medium under horizontal compression in a gravity field are derived. The results are discussed specifying the effects of hydrostatic, deviatoric and couple stresses upon the characteristic propagation velocities of shear and compression wave modes.

\section{INTRODUCTION}

Biot [1] has indicated that some of the basic properties of anisotropic elastic media are provided by analyzing a laminated medium superposed of thin adhering layers which are alternately hard and soft. He also suggested that an equivalent continuous anisotropic elastic medium can approximately be used to provide useful insight into some of the basic features of the statical or dynamical problem of elasticity. The validity of such an approximation is based upon the fact that rigidity contrast of the layers is not too large, and that the thickness of the layers remains sufficiently 
small with respect to the wavelength of the displacement field. Such approximation depends also on the type of problem considered and in many cases it requires some additional refinements.

In a classic work on the theory of deformation of anisotropic elastic solids, Biot [2] suggested that a skin effect is associated with anisotropy. He showed that near a free surface or a surface of discontinuity certain components of the stress field vary rapidly from zero to a maximum value within a thin skin. The concentration of stress also occurs where certain stress components are amplified with the skin thickness. In order to make an additional refinement of his theory, Biot [3] has proposed the effect of couple stresses in elasticity and viscoelasticity of an initially stressed anisotropic solids. He showed that this theory is intended to provide an approximate continuous model valid over a wide range for the mechanics of layered laminated media. This theory when applied to a wide variety of geological structures provides remarkably simple and useful results, and hence can be used for a better understanding of the involved physical features.

When a laminated medium of compressible material is replaced by a continuous medium of anisotropic properties, Biot's [1] analysis gives the composite elastic coefficients. This study is found to be somewhat similar to that used by Postma [4], and also to that considered by Helbig [5] in the theory of acoustic propagation in a laminated medium composed of layers of isotropic materials and is initially stress free. The laminated medium is obtained by superposition of thin adherent layers which are assumed to be alternately hard and soft. In these studies, Biot [1] obtained elastic coefficients in terms of the unknown individual material coefficients after deformation. Recently, Tolstoy [6] has made some slight modifications of Biot's formulation and then obtained simple explicit solutions of the dynamical equations for a prestressed isotropic homogeneous solid under horizontal compression in a gravitational field.

In spite of the above progress, several problems concerning the propagation of elastic waves in a thinly-layered laminated medium with stress couples under initial stresses remain fully or partially understood. This paper is intended to address some of these problems.

The main purpose of this paper is to discuss how to obtain the composite elastic coefficients after deformation in terms of Lame's constants and prestress components of the individual layers before deformation. After determination of such coefficients, an attempt is made to find the explicit solutions of the dynamical equations 
for a pre stressed thinly-layered laminated medium under horizontal compression in a gravitational field. The bending rigidity of the laminated medium is also taken into account by introducing stress couples.

\section{BASIC ASSUMPTIONS AND STRESS-STRAIN RELATIONS}

We consider a thinly laminated medium composed of thin adhering layers which are alternately hard and soft. These hard and soft materials occupy, respectively, fractions $\alpha_{1}$ and $\alpha_{2}$ of the total thickness. If the rigidity contrast of the layers is not too large, and if the layer thickness remains sufficiently small with respect to the wavelength of the deformation field, then such a layered medium behaves approximately like an elastic continuum with anisotropic properties even though the individual layers may be isotropic.

We consider the case of a compressible material. The stress-strain relations for the composite medium are

$$
\begin{aligned}
& t_{11}=c_{11} e_{x x}+c_{12} e_{y y} \\
& t_{22}=c_{12} e_{x x}+c_{22} e_{y y} \\
& t_{12}=2 L e_{e y}
\end{aligned}
$$

where $L$ is the composite slide modulus. Due to compressibility the strain component $e_{y y}$ is not the same in each layer. However, $e_{x x}$ and $t_{22}$ remain the same in both materials. The normal stresses in the hard material and (Biot [1], page 190),

$$
\begin{aligned}
& t_{11}^{(1)}=a_{1} e_{x x}+b_{1} e_{y y}^{(1)} \\
& t_{22}=b_{1} e_{x x}+c_{1} e_{y y}^{(1)}
\end{aligned}
$$

For the soft material, the normal stress components are

$$
\begin{aligned}
& t_{11}^{(2)}=a_{2} e_{x x}+b_{2} e_{y y}^{(2)} \\
& t_{22}=b_{2} e_{x x}+c_{2} e_{y y}^{(2)}
\end{aligned}
$$


The average stress $e_{y y}$ is

$$
e_{y y}=\alpha_{1} e_{y y}^{(1)}+\alpha_{2} e_{y y}^{(2)}
$$

The average strain $t_{11}$ is

$$
t_{11}=\alpha_{1} t_{11}^{(1)}+\alpha_{2} t_{11}^{(2)}
$$

The composite elastic coefficients $C_{i j}$ in equations (2.1)-(2.3) now obtained by eliminating the four variables $t_{11}^{(1)}, t_{11}^{(2)}, e_{y y}^{(1)}, e_{y y}^{(2)}$ from the six equations $(2.4)-(2.9)$. They are

$$
\begin{aligned}
& c_{11}=\alpha_{1} a_{1}+\alpha_{2} a_{2}-\frac{\alpha_{1} \alpha_{2}\left(b_{1}-b_{2}\right)^{2}}{\alpha_{1} c_{2}+\alpha_{2} c_{1}} \\
& c_{12}=\frac{\alpha_{1} b_{1} c_{2}+\alpha_{2} b_{2} c_{1}}{\alpha_{1} c_{2}+\alpha_{2} c_{1}}, \quad c_{22}=\frac{c_{1} c_{2}}{\alpha_{1} c_{2}+\alpha_{2} c_{1}}
\end{aligned}
$$

and

$$
L=1 /\left(\frac{\alpha_{1}}{L_{1}}+\frac{\alpha_{2}}{L_{2}}\right)
$$

For convenience we also define the alternative stress components $t_{i j}$ (Biot [1], page 61)

$$
\begin{aligned}
& t_{11}=s_{11}+s_{11} e_{y y}-s_{12} e_{x y} \\
& t_{22}=s_{22}+s_{22} e_{x x}-s_{12} e_{x y} \\
& t_{12}=s_{12}+\frac{1}{2} s_{12}\left(e_{x x}+e_{y y}\right)-\frac{1}{2}\left(s_{11}+s_{22}\right) e_{x y}
\end{aligned}
$$

3. THE HARD AND SOFT INITIALLY ISOTROPIC MATERIALS

We assume that the acceleration due to gravity points downward and the medium lies in the space $y<0$. The external gravitational field has the components $(0,-g, 0)$.

The principal initial stress components are

$$
\begin{aligned}
& S_{11}^{1}=-P_{1}+\rho g y \\
& s_{22}^{1}=\rho_{1} g y
\end{aligned}
$$


The relations between initial stress and initial strain give

$$
\begin{aligned}
& \varepsilon_{11}^{1}=\frac{\left(\lambda_{1}+2 \mu_{1}\right) S_{11}^{1}-\lambda_{1} S_{22}^{1}}{4 \mu_{1}\left(\lambda_{1}+\mu_{1}\right)} \\
& \varepsilon_{22}^{1}=\frac{\left(\lambda_{1}+2 \mu_{1}\right) S_{22}^{1}-\lambda_{1} S_{11}^{1}}{4 \mu_{1}\left(\lambda_{1}+\mu_{1}\right)}
\end{aligned}
$$

The existence of initial stress introduces anisotropy defined by the elastic coefficients $B_{i j}^{1}(>2)$. These elastic coefficients will be functions of the Lame constants $\lambda_{1}, \mu_{1}$ of the isotropic unstressed state and the prestrains $\varepsilon_{i j}^{1}$. In linear approximation following Biot (Ref. [1], page 111) we can write

$$
\begin{aligned}
& B_{11}^{1}=\left(2 \mu_{1}+\lambda_{1}\right)\left(1+\varepsilon_{11}^{1}-\varepsilon_{22}^{1}\right) \\
& B_{22}^{1}=\left(2 \mu_{1}+\lambda_{1}\right)\left(1+\varepsilon_{22}^{1}-\varepsilon_{11}^{1}\right) \\
& B_{12}^{1}=\lambda_{1}-S_{11}^{1} \\
& Q_{1}=\mu_{1}+\frac{1}{2}\left(\lambda_{1}+\mu_{1}\right) \varepsilon_{11}^{1}+\frac{1}{2}\left(\lambda_{1}+\mu_{1}\right) \varepsilon_{22}^{1}
\end{aligned}
$$

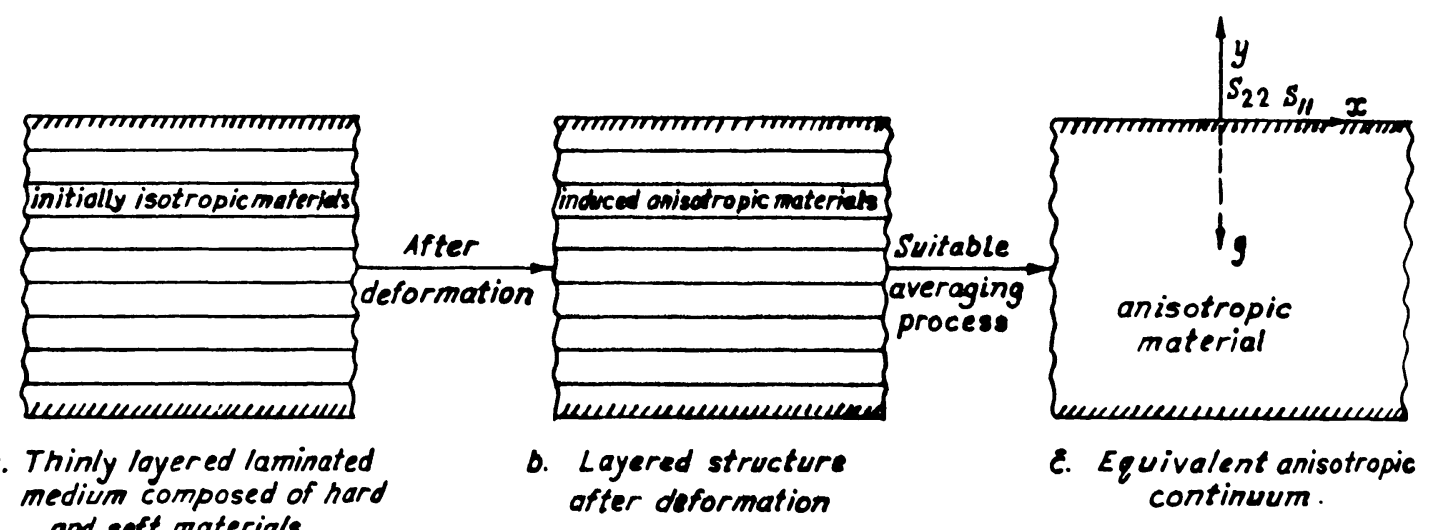

Fig. 1 : Geometry of the problem.

Using (3.2ab), equations (3.3)-(3.6) become

$$
\begin{aligned}
& B_{11}^{1}=\left(2 \mu_{1}+\lambda_{1}\right)\left(1+\frac{S_{11}^{1}-S_{22}^{1}}{2 \mu_{1}}\right) \\
& B_{22}^{1}=\left(2 \mu_{1}+\lambda_{1}\right)\left(1+\frac{S_{22}^{1}-S_{11}^{1}}{2 \mu_{1}}\right)
\end{aligned}
$$




$$
\begin{aligned}
& B_{12}^{1}=\lambda_{1}-S_{11}^{1} \\
& Q=\mu_{1}+\frac{1}{4}\left(S_{11}^{1}+S_{22}^{1}\right)
\end{aligned}
$$

Also $\quad B_{11}^{1}=C_{11}^{1}, \quad B_{12}^{1}=C_{12}^{1}+p_{1}-\rho_{1} g y$;

$$
\begin{aligned}
& B_{21}^{1}=C_{12}^{1}-\rho_{1 g y}=B_{12}^{1}-P_{1} \\
& C_{22}^{1}=B_{22}^{1}, \quad Q^{1}=L_{1}-\frac{1}{4} P_{1}+\frac{1}{2} \rho_{1} g y
\end{aligned}
$$

or

$$
\begin{aligned}
& B_{11}^{1}=a_{1}, \quad B_{12}^{1}=b_{1}+P_{1}-\rho_{1} g y \\
& B_{21}^{1}=b_{1}-\rho_{1} g y=B_{12}^{1}-P_{1} \\
& B_{22}^{1}=c_{1}, \quad A^{1}=L_{1}-\frac{1}{4} P_{1}+\frac{1}{2} \rho_{1} g y
\end{aligned}
$$

Hence, from $(3.1 a b),(3.7)-(3.10)$, and $(3.12 a b c)$, it follows that

$$
\begin{aligned}
& a_{1}=\left(\lambda_{1}+2 \mu_{1}\right)\left(1-\frac{p_{1}}{2 \mu_{1}}\right), \\
& b_{1}=\lambda_{1} \\
& c_{1}=\left(2 \mu_{1}+\lambda_{1}\right)\left(1+\frac{p_{1}}{2 \mu_{1}}\right) \\
& L_{1}=\mu_{1}
\end{aligned}
$$

Following exactly the above derivation the elastic coefficients for the soft material are obtained as

$$
\begin{aligned}
& a_{2}=\left(\lambda_{2}+2 \mu_{2}\right)\left(1-\frac{P_{2}}{2 \mu_{2}}\right) \\
& b_{2}=\lambda_{2} \\
& c_{2}=\left(\lambda_{2}+2 \mu_{2}\right)\left(1+\frac{P_{2}}{2 \mu_{2}}\right) \\
& L_{2}=\mu_{2}
\end{aligned}
$$


4. THE COMPOSITE ANISOTROPIC MATERIAL

Using relations $(2.10)-(2.11 a b),(3.13 a b c d)$ and $(3.14 a b c d)$ we obtain the composite elastic coefficients

$$
\begin{gathered}
C_{11}=\alpha_{1}\left(\lambda_{1}+2 \mu_{1}\right)\left(1-\frac{P_{1}}{2 \mu_{1}}\right)+\alpha_{2}\left(\lambda_{2}+2 \mu_{2}\right)\left(1-\frac{P_{2}}{2 \mu_{2}}\right) \\
-\frac{\alpha_{1} \alpha_{2}}{K}\left(\lambda_{1}-\lambda_{2}\right)^{2} \\
C_{12}=\frac{1}{K}\left[\left(\lambda_{2}+2 \mu_{2}\right)\left(1+\frac{P_{2}}{2 \mu_{2}}\right) \alpha_{1} \lambda_{1}+\left(2 \mu_{1}+\lambda_{1}\right)\left(1+\frac{P_{1}}{2 \mu_{1}}\right) \alpha_{2} \lambda_{2}\right] \\
C_{22}=\frac{1}{K}\left[\left(\lambda_{1}+2 \mu_{1}\right)\left(\lambda_{2}+2 \mu_{2}\right)\left(1+\frac{P_{1}}{2 \mu_{1}}\right)\left(1+\frac{P_{2}}{2 \mu_{2}}\right)\right] \\
L=1 /\left(\frac{\alpha}{\mu_{1}}+\frac{\alpha_{2}}{\mu_{2}}\right)
\end{gathered}
$$

and

$$
K=\alpha_{1}\left(\lambda_{2}+2 \mu_{2}\right)\left(1+\frac{P_{2}}{2 \mu_{2}}\right)+\alpha_{2}\left(\lambda_{1}+2 \mu_{1}\right)\left(1+\frac{P_{1}}{2 \mu_{1}}\right)
$$

5. FORMULATION OF THE PROBLEM FOR THE COMPOSITE MATERIAL

The average initial stresses in the $x$ and $y$ directions are

$$
\begin{aligned}
& S_{11}=\alpha_{1} S_{11}^{1}+\alpha_{2} S_{11}^{2}=-p+\rho g y \\
& S_{22}=\alpha_{1} S_{22}^{1}+\alpha_{2} S_{22}^{2}=\rho g y
\end{aligned}
$$

where $P=\alpha_{1} P_{1}+\alpha_{2} P_{2}$ and $\rho=\alpha_{1} \rho_{1}+\alpha_{2} \rho_{2}$

It follows from $(2.13)-(2.15)$ that

$$
\begin{aligned}
& t_{11}=s_{11}+(-p+\rho g y) e_{y y} \\
& t_{22}=s_{22}+\rho g y e_{x x} \\
& t_{12}=s_{12}-\left(-\frac{1}{2} p+\rho g y\right) e_{x y}
\end{aligned}
$$

From (2.1)-(2.3) and 5.3abc) equating t's we get after simplification, 


$$
\begin{aligned}
& s_{11}=B_{11} e_{x x}+B_{12} e_{y y} \\
& S_{22}=B_{21} e_{x x}+B_{22} e_{y y} \\
& S_{12}=20 e_{x y}
\end{aligned}
$$

where $\quad B_{11}=C_{11}, \quad B_{12}=C_{12}+P-\rho g y$

$$
\begin{aligned}
& B_{21}=C_{12}-\rho g y=B_{12}-P \\
& B_{22}=C_{22}, \quad Q=L-\frac{1}{4} P+\frac{1}{2} \rho g y
\end{aligned}
$$

where C's are given by (4.1abcde).

\section{COUPLE STRESS ANALOGY}

If the hard layer is sufficiently stiff a couple stress of moment $M$ per unit area is produced in the plane normal to the $x$ axis. In this case $t_{12} \neq t_{21}$. Equilibrium of moments for an element of material requires the condition

$$
T_{12}-t_{21}=\frac{\partial M}{\partial x}
$$

If we now introduce the effect of couple stress, the equilibrium equations are obtained as (Biot [3])

$$
\begin{aligned}
& \frac{\partial t_{11}}{\partial x}+\frac{\partial t_{12}}{\partial y}=\rho \frac{\partial^{2} u}{\partial t^{2}} \\
& \frac{\partial t_{12}}{\partial x}+\frac{\partial t_{22}}{\partial y}=\rho \frac{\partial^{2} v}{\partial t^{2}}+P \frac{\partial^{2} v}{\partial x^{2}}+\frac{\partial^{2} M}{\partial x^{2}}
\end{aligned}
$$

The value of the moment $M$ in terms of the deformation is obtained as (Biot [3])

$$
M=b \frac{\partial^{2} v}{\partial x^{2}}
$$

where the couple stress coefficient is

$$
b=\frac{1}{3} h^{2} \alpha_{1} \alpha_{2} \frac{\left(L_{1}-L_{2}\right)}{\left(\alpha_{1} L_{1}+\alpha_{2} L_{2}\right)}\left(M_{1} \alpha_{1}-M_{2} \alpha_{2}\right)
$$


with $h=h_{1}+h_{2}$.

$$
\begin{aligned}
M_{i} & =\frac{1}{4 C_{i}}\left(a_{i} c_{i}-b_{i}^{2}\right) \\
& =\frac{1}{4\left(\lambda_{i}+2 \mu_{i}\right)\left(1+\frac{i}{2 \mu_{i}}\right)}\left[\left(\lambda_{i}+2 \mu_{i}\right)^{2}\left(1-\frac{P_{i}^{2}}{4 \mu_{i}^{2}}\right)-\lambda_{i}^{2}\right], \quad i=1,2 .
\end{aligned}
$$

When one layer is much more rigid than the other the couple stress coefficient has the simple value

$$
b=\frac{1}{3} h^{2} M \quad \text { with } \quad M=M_{1} \alpha_{1}
$$

7. GOVERNING DYNAMICAL EQUATIONS AND THEIR SOLUTIONS

In view of $(5.3 a b c)-(5.4 a b c)$, equations $(6.2)-(6.3)$ become

$$
\begin{aligned}
& C_{11} \frac{\partial^{2} u}{\partial x^{2}}+B \frac{\partial^{2} v}{\partial x \partial y}+L \frac{\partial^{2} u}{\partial y^{2}}=\rho \frac{\partial^{2} u}{\partial t^{2}} \\
& C_{22} \frac{\partial^{2} v}{\partial y^{2}}+B \frac{\partial^{2} v}{\partial x \partial y}+(L-P) \frac{\partial^{2} v}{\partial x^{2}}=\rho \frac{\partial^{2} v}{\partial t^{2}}+b \frac{\partial^{4} v}{\partial x^{4}} .
\end{aligned}
$$

where $B=C_{12}+L$ and $L=Q+\frac{P}{4}-\frac{1}{2}$ gpy

It is noted that the effect of the buoyancy terms in equations (7.1)-(7.2) is appreciably smaller than that of pre-stressed values for a period of half a minute or less. In dealing with seismic wave problems, we can neglect the buoyancy terms in (7.1)-(7.2) compared with the pre-stressed terms. Using now (5.5abc) and neglecting the buoyancy terms in the above equations, it turns out that

$$
\begin{aligned}
& B_{11} \frac{\partial^{2} u}{\partial x^{2}}+\left(B_{12}-\frac{3}{4} P+Q\right) \frac{\partial^{2} v}{\partial x \partial y}+\left(Q+\frac{1}{4} P\right) \frac{\partial^{2} u}{\partial y^{2}}=\rho \frac{\partial^{2} u}{\partial t^{2}} \\
& B_{22} \frac{\partial^{2} v}{\partial y^{2}}+\left(Q-\frac{3}{4} P\right) \frac{\partial^{2} v}{\partial x^{2}}+\left(B_{12}+Q-\frac{3}{4} P\right) \frac{\partial^{2} u}{\partial x \partial y}=\rho \frac{\partial^{2} v}{\partial t^{2}}+b \frac{\partial^{4} v}{\partial x^{4}}
\end{aligned}
$$

we next introduce the classic potentials $\phi, \psi$ defined by

$$
u=\phi_{x}-\psi_{y} \text {, and } v=\phi_{y}+\psi_{x}
$$




$$
\begin{aligned}
& B_{11}\left(\phi_{x x x}-\psi_{y x x}\right)+\left(B_{12}-\frac{3}{4} P+Q\right)\left(\phi_{y y x}+\psi_{x x y}\right) \\
& +\left(Q+\frac{1}{4} P\right)\left(\phi_{x y y}-\psi_{y y y}\right)=\rho\left(\phi_{y t t}-\psi_{y t t}\right) \\
& B_{12}\left(\phi_{y y y}+\psi_{x y y}\right)+\left(Q-\frac{3}{4} P\right)\left(\phi_{y x x}+\psi_{x x x}\right)+\left(B_{12}+Q-\frac{3}{4} P\right)\left(\phi_{x x y}-\psi_{y y x}\right) \\
& =\rho\left(\phi_{y t t}+\psi_{x t t}\right)+b\left(\phi_{y x x x x}+\psi_{x x x x x}\right)
\end{aligned}
$$

For some interesting solutions we take the trial functions as

$$
\begin{aligned}
& \phi=\phi\left(a_{1} x+B_{1} y \pm c_{1} t\right) \\
& \psi=\psi\left(a_{2} x+b_{2} y \pm c_{2} t\right)
\end{aligned}
$$

Assuming now a pure shear wave travelling in the $x$-direction, we set

$$
\phi=0, \quad a_{2}=1, \quad b_{2}=0, \quad \psi=\psi\left(x \pm c_{2} t\right)
$$

Then

$$
u=0, \quad v=\psi_{x}
$$

and

$$
\frac{\psi^{\prime \prime \prime \prime}}{\psi+1 T T}=\left(Q-\frac{3}{4} P-\rho c_{2}^{2}\right) / b
$$

Thus when the functional form of $\psi$ is known then (7.13) corresponds to a shear mode of the SV type $(u=0)$ propagating in the $S_{11}$ i.e. $x$-direction, with velocity $c_{2}$.

In general, for plane harmonic waves along the $x$-direction, we write

$$
\psi=\exp \left[i\left(x \pm c_{2} t\right)\right]
$$

Then, from equation (7.13), we obtain

$$
c_{s x}^{2}=\left(Q+b-\frac{3}{4} P\right) / \rho
$$

Evidently, the shear wave velocity, $\mathrm{c}_{\mathrm{sx}}$ depends on the initial stress, couple stress and hydrostatic stress. 
Likewise a shear wave travelling in the vertical $y$-direction corresponds to

$$
\phi=0, \quad \partial_{2}=0, \quad b_{2}=1, \quad \psi\left(y \pm c_{2} t\right)
$$

Then it follows from $(7.7)-(7.8)$ that the shear wave velocity is

$$
c_{s y}^{2}=(Q+P / 4) / p
$$

In this case of shear waves travelling in the $y$-direction, there is no effect of couple stress.

We also note

$$
c_{s y}^{2}-c_{s x}^{2}=c_{s}^{2}(P-b) / Q
$$

For pure compressional modes, we take

$$
\psi=0, \quad a_{1}=1, \quad b_{1}=0, \quad \phi=\phi\left(x \pm c_{1} t\right)
$$

so that the wave velocity is

$$
c_{p x}^{2}=B_{11} / \rho
$$

In the case of harmonic waves the velocity also remains the same.

Likewise there exists a pure P-mode travelling in the vertical direction,

$$
\psi=0, \quad a_{1}=0, \quad b_{1}=1, \quad \phi=\phi\left(y \pm c_{1} t\right)
$$

In view of equation (7.8), we have

$$
c_{p y}^{2}=B_{22} / \rho
$$

and hence

$$
c_{p y}^{2}-c_{p x}^{2}=\frac{B_{22}-B_{11}}{\rho}
$$


As in the case of a single isotropic layer (Tolstoy [6]) there are however, apart from the principal axes of pre-stress, two further conjugate directions for which uncoupled i.e. pure modes of shear of the pre-stress field occur.

We assume

$$
\psi=0, \quad \phi=\phi\left(a_{1} x+b_{1} y \pm c_{1} t\right),
$$

where

$$
a_{1}=\sin \theta, \quad b_{1}=\cos \theta
$$

It turns out that

$$
c_{1}^{2}=\frac{B_{11} a_{1}^{2}+\left(B_{12}-P / 2+2 Q\right) b_{1}^{2}}{\rho}
$$

and

$$
\tan ^{2} \theta=\frac{\left.-(m+n)+\left[(m-n)^{2}+4 m r\right)\right]^{1 / 2}}{2}
$$

where

$$
m=\left(B_{22}-B_{12}-2 Q+P / 2\right), \quad n=\left(B_{12}+2 Q-\frac{3}{2} P-B_{11}\right), \quad r=\frac{b}{\phi}
$$

A similar uncoupled solution exists for shear modes. We assume the solutions as

$$
\phi=0, \quad \psi=\psi\left(a_{2} x+b_{2} \pm c_{2} t\right)
$$

where

$$
a_{2}=\sin \theta . \quad b_{2}=\cos \theta
$$

Setting

$$
\psi=e^{i\left(a_{2} x+b_{2} y \pm c\right.} c^{t)}, \quad a_{2}=\sin \theta, \quad b_{2}=\cos \theta
$$

We obtain

$$
c_{2}^{2}=\frac{a_{2}^{2}\left(B_{11}-B_{12}+\frac{3}{4} P-Q\right)+\left(Q+\frac{P}{4}\right) b_{2}^{2}}{\rho}
$$




$$
\tan ^{2} \theta=\frac{-B_{1}+\left(B_{1}^{2}-4 A_{1} A_{2}\right)^{1 / 2}}{2 A_{2}}
$$

where

$A_{1}=B_{22}-B_{12}-2 Q+\frac{1}{2} P, B_{1}=B_{22}-B_{11}-P, A_{2}=B_{12}-B_{11}+b+2 Q-\frac{3}{2} P$ (7.30abc)

Thus we obtain explicit expressions for the effects of hydrostatic stress, deviatoric stress and couple stress upon the characteristic propagation velocities of shear and compression modes by using equations $(7.15),(7.17),(7.20)$ and $(7.21)$ with relations (4.1) and $(5.5 \mathrm{abc})$;

$$
\begin{aligned}
& c_{s x}^{2}=\frac{1}{\rho}\left(L+b-P+\frac{1}{2} \rho g y\right) \\
& c_{s y}^{2}=\frac{1}{\rho}\left(L+\frac{1}{2} y \rho g\right) \\
& c_{p x}^{2}=\frac{1}{\rho}\left[\alpha_{1}\left(\lambda_{1}+2 \mu_{1}\right)\left(1-\frac{P_{1}}{2 \mu_{1}}\right)+\alpha_{2}\left(\lambda_{2}+2 \mu_{2}\right)\left(1-\frac{P_{2}}{2 \mu_{2}}\right)-\frac{\alpha_{1} \alpha_{2}}{K}\left(\lambda_{1}-\lambda_{2}\right)^{2}\right] \\
& c_{p y}^{2}=\frac{1}{K \rho}\left[\left(\lambda_{1}+2 \mu_{1}\right)\left(\lambda_{2}+2 \mu_{2}\right)\left(1+\frac{P_{1}}{2 \mu_{1}}\right)\left(1+\frac{P_{2}}{2 \mu_{2}}\right)\right] \\
& \text { where } \quad K=\alpha_{1}\left(\lambda_{2}+2 \mu_{2}\right)\left(1+\frac{P_{2}}{2 \mu_{2}}\right)+\alpha_{2}\left(\lambda_{1}+2 \mu_{2}\right)\left(1+\frac{P_{1}}{2 \mu_{1}}\right)
\end{aligned}
$$

8. SUMMARY AND CONCLUSION

The theory presented in this paper enables us to determine the composite elastic coefficients $c_{i j}$. These coefficients were not known earlier for a laminated structure. After deformation, the state of initial stresses (different for hard and soft materials) introduces anisotropy. The corresponding elastic coefficients $B_{i j}$ are obtained in section 3 . When $B_{i j}$ are known for two different materials, it is also possible to find out the corresponding $C_{i j}$ 's through equations (3.1labc). On the other hand, when $C_{i j}$ 's are known for two different materials, then Biot's (1, p. 190] analysis can be used to obtain the composite elastic coefficients.

In some cases, the use of the equivalent anisotropic continuum with elastic coefficients in equations $(4.1 a)-(4.1 e)$ are not sufficient. So the bending rigidity of the laminations are taken into account by introducing stress couples through 
equations (4.11)-(4.12) as stated by Biot [3]. The corresponding equilibrium equations are obtained in section 6 .

Based upon the equilibrium equations for the composite elastic material and using an analysis similar to Tolstoy [6], the velocities of the $P$ and SV wave modes are obtained explicitly.

The effects of couple stresses and initial stresses are found to be large for values approaching the instability value. The effect on the propagation velocity can then be quite large. As for the effects of gravity, these seem to be sensible for a very weak (low rigidity) sediments and for a very low frequency at which buoyancy effects are significant. This point deserves further attention and will be discussed in a subsequent paper.

\section{CONCLUDING REMARKS}

Although the present theory is developed in the context of plane strain deformation, it can be extended to three-dimensional problems of transverse isotropic materials with rectangular, triangular and circular plane form. Also, further extension of results is possible for a thermoclastic laminated medium. Such problems will be treated in a subsequent paper.

ACKNOWLEDGEMENT. This work was partially supported by the University of Central Florida. This work was done during the first author's tenure as an honorary postdoctoral fellow at the Department of Applied Mathematics, University of Calcutta, India.

\section{REFERENCES}

1. BIOT, M. A. Mechanics of Incremental Deformations, John Wiley and Sons (1965).

2. BIOT, M. A. Fundamental Skin Effect in Anisotropic Solid Mechanics, Internat. J. Solid Struct., 2 (1986), 645-663.

3. BIOT, M. A. Buckling and Dynamics of Multilayered and Laminated Plates Under Initial Stress, Int. J. Solids Struct. 10 (1974), 419-451.

4. POSTMA, G. W. Wave Propagation in a Stratified Medium, Geophysics, 20 (1955), 780-806.

5. HELBIG, K. Elastische Wellen in Aisotropen Medien, Gerlands Beitrage Zur Geophysik, 67 (1958), 256-288.

6. TOLSTOY, I. On Elastic Waves in Prestressed Solids, J. Geoph. Research 87 $(1982), 6823-6827$. 


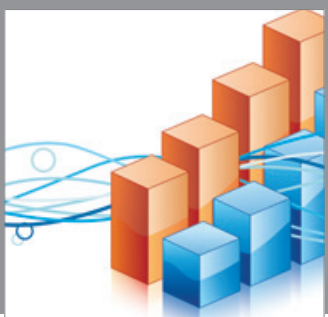

Advances in

Operations Research

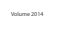

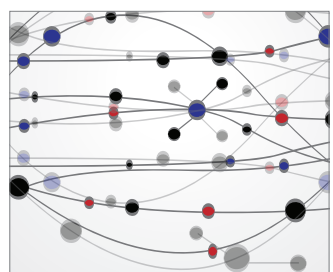

\section{The Scientific} World Journal
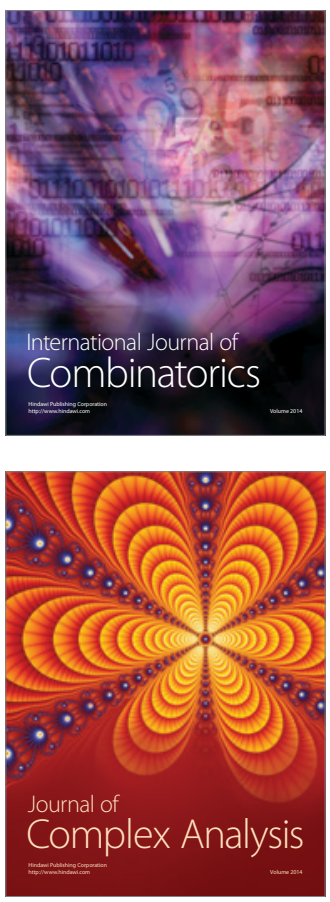

International Journal of

Mathematics and

Mathematical

Sciences
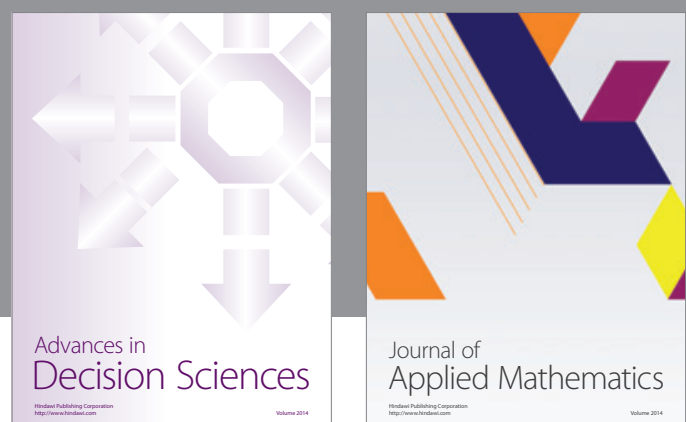

Journal of

Applied Mathematics
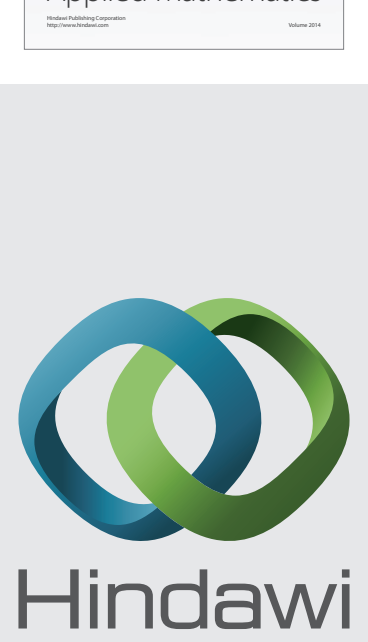

Submit your manuscripts at http://www.hindawi.com
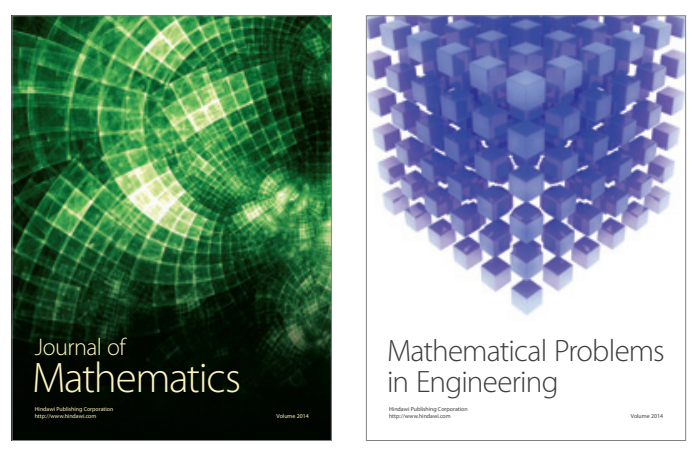

Mathematical Problems in Engineering
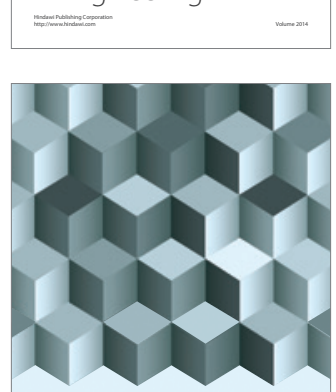

Journal of

Function Spaces
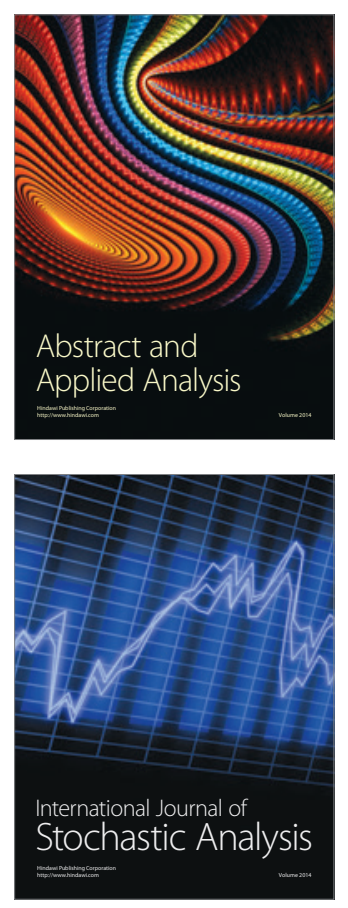

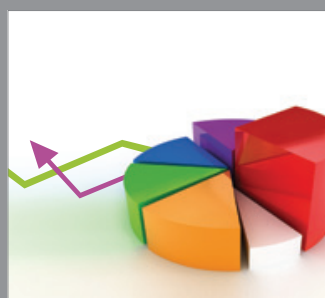

ournal of

Probability and Statistics

Promensencen
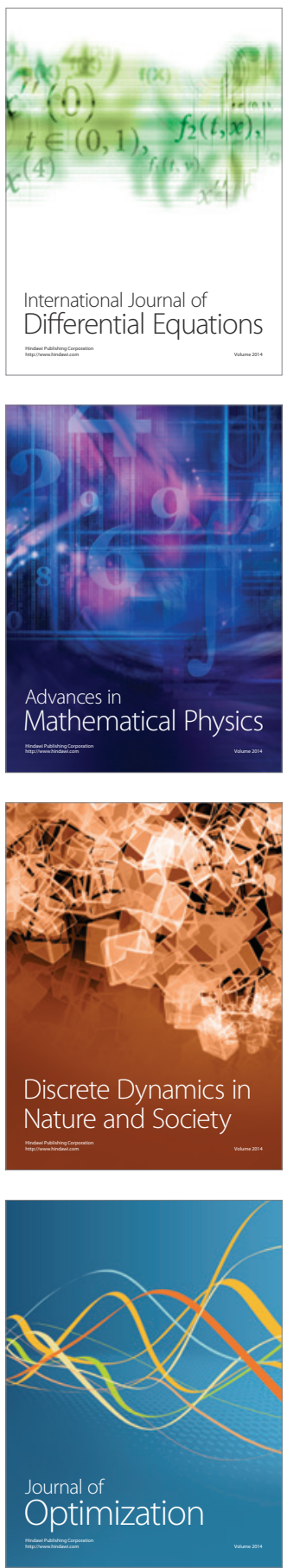